Bull. Mater. Sci., Vol. 22, No. 2, April 1999, pp. 109-113. (C) Indian Academy of Sciences.

\title{
Open- and short-circuit thermally stimulated currents in ethyl cellulose (EC) : polymethyl methacrylate (PMMA) blend
}

\author{
P K KHARE*, J M KELLER and S C DATT \\ Department of Postgraduate Studies and Research in Physics, Rani Durgavati University, Jabalpur 482 001, India
}

MS received 6 April 1998; revised 7 January 1999

\begin{abstract}
Mechanisms of charge generation and its persistence in one and both-side vacuum-aluminized ethyl cellulose (EC) : polymethyl methacrylate (PMMA) blend thermoelectrets, prepared under different fields $(10,25,50$ and $100 \mathrm{kV} / \mathrm{cm})$ and temperatures $\left(40,60,80\right.$ and $\left.100^{\circ} \mathrm{C}\right)$, have been analysed using short- and open-circuit thermally stimulated depolarization current (TSDC) technique. The TSDCs were recorded by reheating the samples at a linear heating rate of $4^{\circ} \mathrm{C} / \mathrm{min}$. The TSDC thermograms of polyblends containing EC : PMMA in different weight ratio are, in general, characterized with two peaks in lower and higher temperature regions. However, the polarity of the peaks was found to be just opposite in short- and open-circuit TSDC measurements. Moreover, results on $97: 3,93: 7$ and $90: 10$ EC: PMMA polyblends indicated that the current increases with concentration of PMMA. The results indicate the existence of heterocharge due to dipole orientation and ionic charge drift together with the injection of charge carriers from electrodes with their subsequent localization in surface and bulk traps. Further, the chances of charge trapping in polyblends, at the interfaces are greater than in the individual polymers.
\end{abstract}

Keywords. Thermally stimulated currents; heterocharge; homocharge; plasticization efiect; induced dipoles.

\section{Introduction}

A rapid growth of interest, in recent years, in studying charge storage and transport phenomena in polymer electrets by means of techniques based on thermal stimulation has been indicated by a number of publications (Shrivastava et al 1979, 1981; Pillai et al 1981; Khare et al 1992, 1996). The thermally stimulated depolarization current (TSDC) is a powerful technique that has provided valuable information on the charge storage and transport behaviour of many polymers (Sinha et al 1981; Keller et al 1993; Khare 1994; Khare et al 1994a, b). The technique has shown that for improving charge storage properties of polymers and obtaining strong and stable electrets, a better understanding of the structural or morphological details and dynamical properties of the polymers is required on both molecular and super molecular levels (Frensch and Wendorff 1986). It has been reported that the electret state in a polymer can be produced not only by conventional methods, but also by bringing about some structural changes in the electret forming materials (Khare and Singh 1994). Investigations concerning the dependence of electrical properties on structure are, however, presently very sparse. Appropriate model systems are required to obtain definite conclusions.

*Author for correspondence
Multicomponent polymers, specially blends of two chemically different polymers that are partially compatible, comprise such model systems.

Ethyl cellulose is a partially polar polymer having density 1.14 , melting temperature $165-175^{\circ} \mathrm{C}$ and glass transition temperature, $T_{g}, 60-75^{\circ} \mathrm{C}$. Polymethyl methacrylate is strongly polar and mostly amorphous in character, having density 1.88 , melting temperature $180^{\circ} \mathrm{C}$ and glass transition temperature, $T_{\mathrm{g}}, 114^{\circ} \mathrm{C}$. The two polymers thus differ remarkably in their glass transition temperature, i.e. in their dynamical as well as structural properties. Blends of these two polymers are expected to yield strong and long-lived electrets useful for industrial applications.

Several reports on TSDC behaviour of EC and PMMA thermoelectrets and different relaxation processes contributing to the observed peaks in the corresponding thermograms are available (Shrivastava et al 1978; Vanderschueren and Linkens 1978; Jiska et al 1981; Khare and Srivastava 1993, 1994; Khare et al 1993, 1994c). However, the role of various polarization processes and their relative contribution to the electret state of the polymers is not yet fully understood. Particularly, the space charge relaxation mechanism and the details of trap structure (including the trap distribution in energy and also over the volume of the polymer) are still to be well understood. Such information can best be obtained by combined study of open- and short-circuit TSCs. 
In this paper we report the results of open- and short-circuit TSDC measurements carried out on EC : PMMA blends.

\section{Experimental}

EC and PMMA used in the present investigation were obtained from M/s Redox Chemicals, Jabalpur. Isothermal immersion technique was utilized for preparing films. Three blend samples prepared were $97: 3,93: 7,90: 10$ by weight proportion of ethyl cellulose and polymethyl methacrylate, and designated as $P_{1}, P_{2}$ and $P_{3}$, respectively. The solution was prepared in a glass beaker by first dissolving $2.4 \mathrm{~g} \mathrm{EC}$ in $30 \mathrm{ml}$ of chemically pure benzene. Appropriate amount of PMMA was then mixed in it. The solution was continuously stirred for about $30 \mathrm{~min}$ by means of a teflon-coated magnetic stirrer. Thereafter it was stirred and heated up to $60^{\circ} \mathrm{C}$ to yield a homogeneous solution. The solution thus prepared was poured onto clean glass plate floating over the mercury pool and the solvent was allowed to evaporate inside an air oven at $40^{\circ} \mathrm{C}$. The desired samples thus obtained were subjected to room temperature outgassing at a pressure of $10^{-5}$ torr for a period of $12 \mathrm{~h}$ to remove any residual solvent. Such preconditioned samples were then unilaterally and bilaterally vacuum-aluminized over a central circular area of $42 \mathrm{~mm}$ diameter.

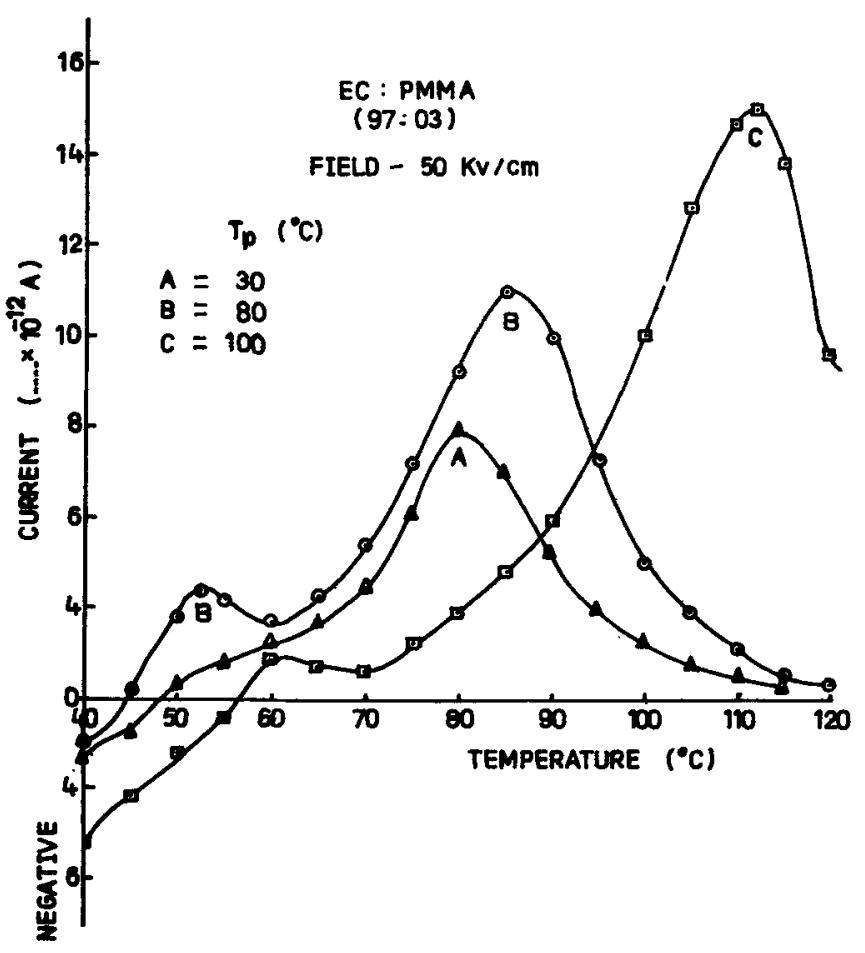

Figure 1. Open-circuit TSC thermograms for EC: PMMA $(97: 3)$ blends polarized at different temperatures (40, 80 and $100^{\circ} \mathrm{C}$ ) with $50 \mathrm{kV} / \mathrm{cm}$ field.
The samples were thermally polarized with fields of $10,25,50$ and $100 \mathrm{kV} / \mathrm{cm}$ at temperatures $40,60,80$ and $100^{\circ} \mathrm{C}$. After polarizing for $1 \mathrm{~h}$ at the desired temperature, the samples were cooled to room temperature under the application of the field in 1-1/2 h. Total time of polarization was thus adjusted to be $2.5 \mathrm{~h}$ in each case. Polarized samples were subsequently short-circuited for an arbitrary time of $5 \mathrm{~min}$ so as to remove the frictional and stray charges present, if any. The short-circuit TSDCs on bilaterally aluminized samples were then recorded by reheating the samples at a linear rate of $4^{\circ} \mathrm{C} / \mathrm{min}$. For registering TSDC in open-circuit, the unilaterally polarized sample was mounted in the electrode assembly with its non-metallized surface parallel to the sensing electrode at a distance of $3 \mathrm{~mm}$, while the metallized surface of the sample rested on the other metal electrode.

A high voltage power supply EC $4800 \mathrm{D}$, provided stabilized DC voltages for polarization while TSDCs were measured with the help of a Keithley $610 \mathrm{C}$ Electrometer.

\section{Results and discussion}

The characteristic open-circuit TSDC thermograms for $P_{1}$ and $P_{3}$ polyblends polarized at various temperatures are shown in figures 1 and 2 , respectively. Initially, the

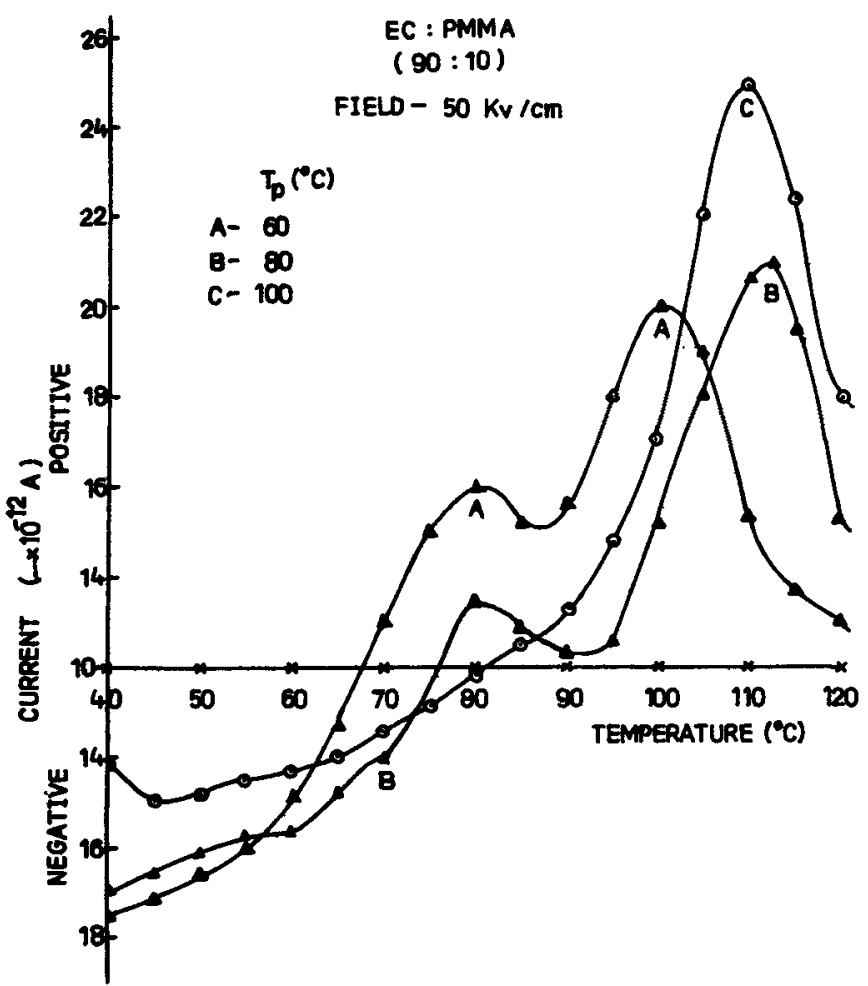

Figure 2. Open-circuit TSC thermograms for EC: PMMA $(90: 10)$ blends polarized at different temperatures $(60,80$ and $100^{\circ} \mathrm{C}$ ) with $50 \mathrm{kV} / \mathrm{cm}$ field. 
current, in general is normal and, flows in a direction opposite to the charging current or is negative; it, however, changes sign and starts flowing in the same direction as the charging current at higher temperatures. Further, the temperature of polarity reversal increases with the increase in poling temperature. The $P_{1}$ blends show a TSDC peak between 50 and $60^{\circ} \mathrm{C}$ (in some cases only a hump is observed) and a second peak between 78 and $115^{\circ} \mathrm{C}$. The $P_{3}$ blends show a low temperature peak between 70 and $80^{\circ} \mathrm{C}$ and the high temperature peak between 95 and $110^{\circ} \mathrm{C}$. The peak temperature shifts towards higher temperature on increasing the polarizing temperature. The temperature of current reversal is also found to increase with increase in forming temperature or the discharge current is found to flow in the negative direction up to a higher temperature for samples polarized at higher temperatures.

Figure 3 shows TSDCs for $P_{2}$ blends polarized at $60^{\circ} \mathrm{C}$ with various fields (i.e. $10,25,50$ and $100 \mathrm{kV} / \mathrm{cm}$ ). The magnitude of the peaks is found to increase considerably with increase in poling field. Further, the high temperature peak shifts towards lower temperature.

Characteristic short-circuit TSDC thermograms for $P_{2}$ blends poled at various temperatures with a poling field of $100 \mathrm{kV} / \mathrm{cm}$ and those poled at $60^{\circ} \mathrm{C}$ with various poling fields are shown in figures 4 and 5 , respectively.

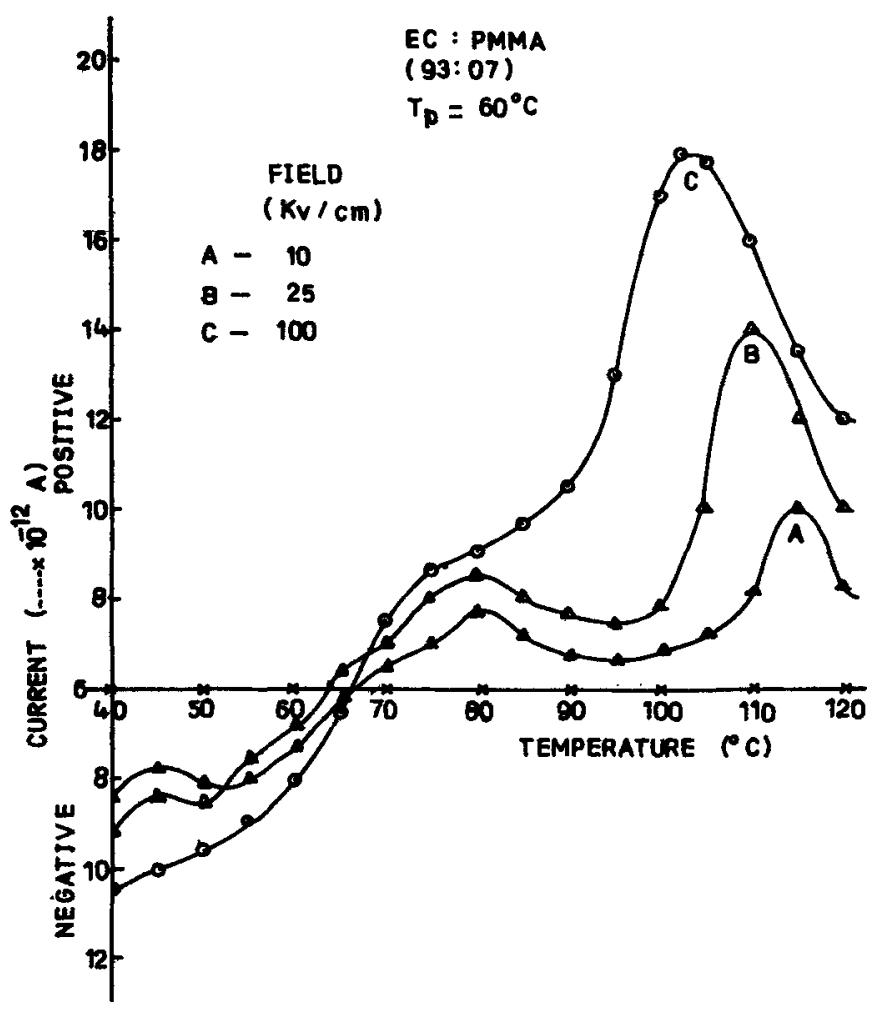

Figure 3. Open-circuit TSC thermograms for EC: PMMA (93: 7) blends polarized at $60^{\circ} \mathrm{C}$ with different fields $(10,25$ and $100^{\circ} \mathrm{C}$ ).
The thermograms, in general, are characterized with two peaks in the temperature range $75-85^{\circ} \mathrm{C}$ and $100-110^{\circ} \mathrm{C}$, respectively, both in the positive direction. However, in some cases, the discharge current flows in the negative direction for some part of TSDC cycle but eventually changes sign and flows in the positive direction for the remaining part of the discharge cycle.

The classical theory (Gubkin 1957) for the decay of charge of an electret, assumes the superposition of homocharge and heterocharge. Homocharge is the space charge consisting of ions and electrons and is produced by the discharge in air gap between poling electrode and sample. Heterocharge, on the other hand, is volume charge produced by either the rotation of dipoles or the separation of ions. Since the total charge is the sum of homocharge and heterocharge, if the observed TSDC is due to dipole disorientation or ion displacement, during heating process, the sign of the discharge current is expected to be of opposite polarity to that of the charging current. On the other hand, if it is due to dissipation of space charges, the sign of the TSDC is expected to be the same as charging current or positive in polarity.

The TSDC thermograms in the present case show the co-existence of homo- and hetero-charge. As exhibited by thermograms of figures 1 and 2 , heterocharge seems to be dominant at low discharge temperatures so that the net current is negative. This results in increase of temperature of reversal. At low temperatures, the electric

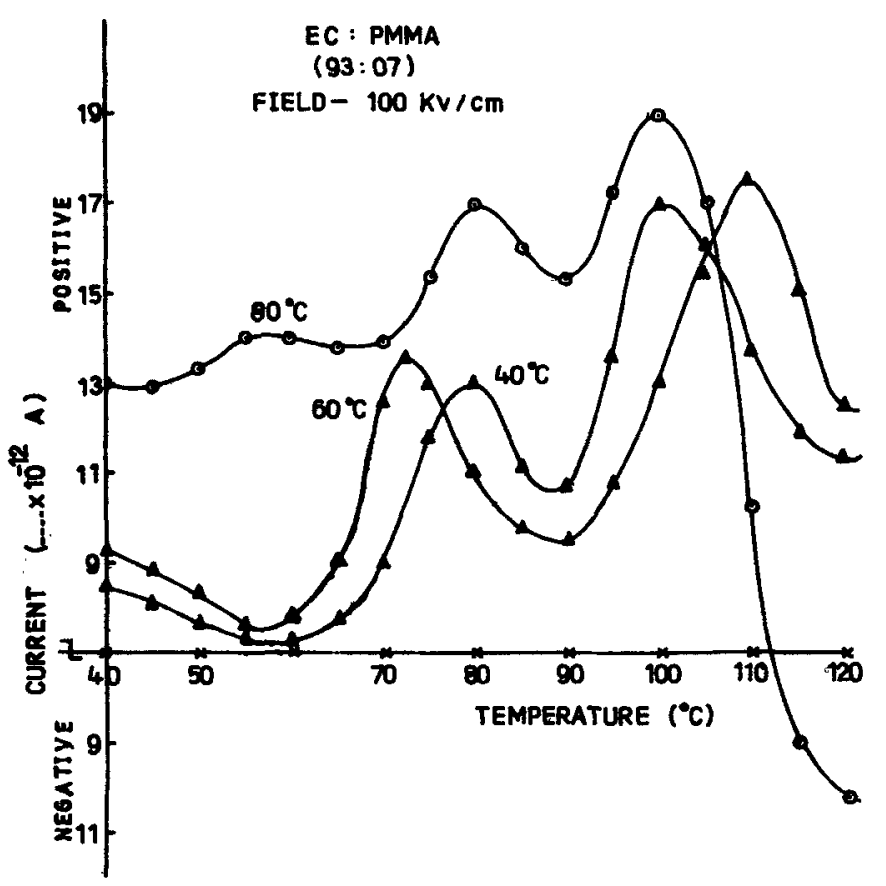

Figure 4. Short-circuit TSC thermograms for EC: PMMA $(93: 7)$ blends polarized at different temperatures $(40,60$ and $\left.80^{\circ} \mathrm{C}\right)$ with a poling field $(100 \mathrm{kV} / \mathrm{cm})$. 
conductivity of the samples is expected to be low and hence cancellation of homocharge ions cannot take place. It is only expected, therefore, that the movement of dipoles is thermally activated at these low temperatures. At higher temperatures, the discharge current is found to correspond to homocharge indicating cancellation of charge carriers forming space charge. Further, high poling temperature seem to favour heterocharge formation or orientation of dipoles and ion separation. Also increase in positive current with increase in poling field indicates increased homocharge formation under high poling field conditions.

In the case of open-circuit TSDC, the charge carriers will not be able to recombine with the image charges at the adjoining electrode due to blocking by adjacent air gap. This blocking will cause these carriers to move towards the other electrode through the bulk of the sample. The motion of these carriers towards the lower electrode will release the charge carriers induced on the coated electrode resulting in a current of positive polarity.

Ethyl cellulose is a weakly polar polymer having glass transition temperatures, $T_{\mathrm{g}}$, between $45-65^{\circ} \mathrm{C}$. PMMA on the other hand, is a strongly polar polymer with $T_{\mathrm{g}}$ around $110^{\circ} \mathrm{C}$. The observed initial heterocharge is, there-

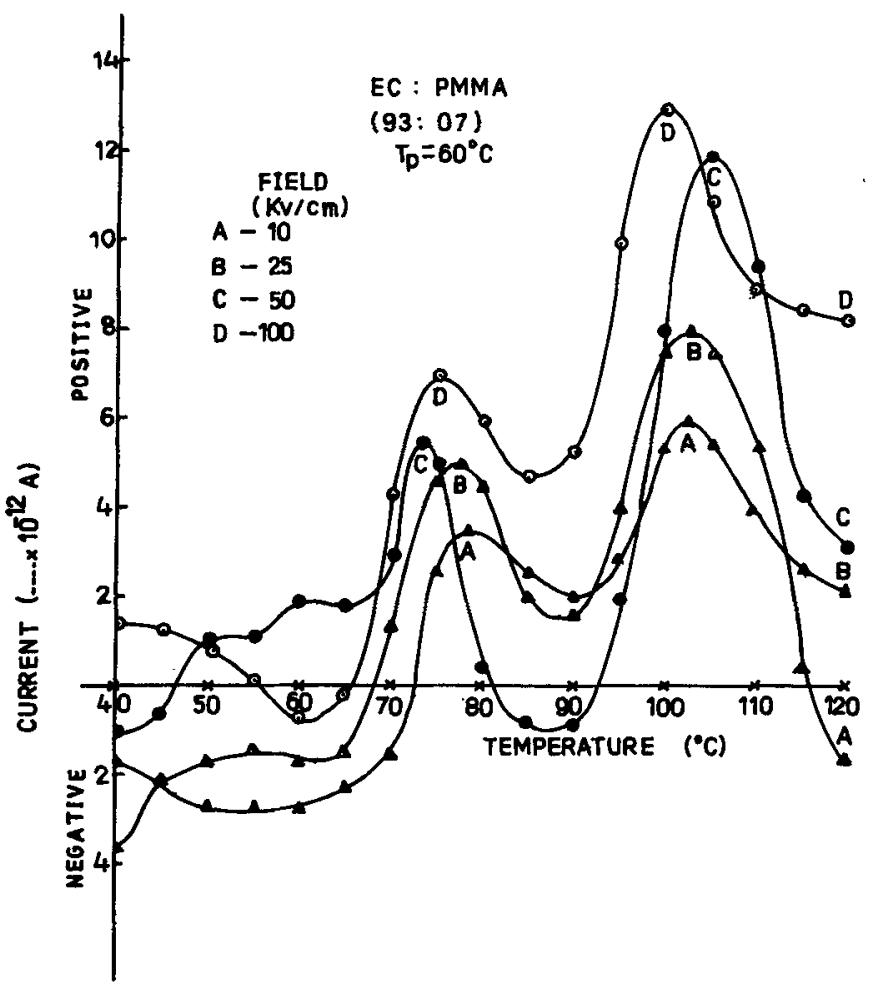

Figure 5. Short-circuit TSC thermograms for EC: PMMA (93:7) blends polarized at $60^{\circ} \mathrm{C}$ with different poling fields $(10,25,50$ and $100 \mathrm{kV} / \mathrm{cm})$. fore, expected to be due to dipole orientations associated with molecular motions near the $T_{p} \mathrm{~s}$ of the two polymers.

Both open-circuit as well as the short-circuit TSDC thermograms, in general, show peaks located around $60-80$ and $95-115^{\circ} \mathrm{C}$, respectively. Polymers are known to contain a large number of traps. During charging, the traps are always successively filled, beginning with the shallow sites. Thus, upon heating the charged sample, the carriers in the shallow level become mobile with free path much shorter than the sample thickness. With the rising temperature, the measured current increases due to the increase in number of available carriers in quasi-conduction band. This explains the initial exponential increase in current. The shift of high temperature peak towards higher temperature with increasing value of poling temperature indicates the trapping of electronic charges in energetically distributed traps existing in the polymer.

Considering the origin of high temperature peak to be mainly due to space charge polarization, the thermograms show that the traps are distributed spatially as well as energetically. The occurrence of well-defined and nonoverlapping peaks, indicates that the polymer contains trapping levels of at least two different depths with certain narrow energy distribution. The activation energy values calculated by the initial rise method for both the peaks range from $0.29-0.38 \mathrm{eV}$ and $0.68-0.73 \mathrm{eV}$.

Increase in discharge current with increased PMMA content, as evident from figures 1 and 2, may be understood in terms of increased charge formation. Because of the inherent heterogeneous structure of the blend charge carriers may pile up at the phase boundaries. In EC : PMMA matrices containing different percentage of PMMA, at least two phases co-exist and discontinuity of structure at such boundary increases. Because of the difference of carrier conductivity of different phases, the carriers get trapped at the interphases of phase boundary. Further, trapping of both positive and negative charge carriers in the various traps may also result in formation of induced dipoles. In fact, the observed value of activation energy may be considered as an indication of induced dipole formation because in this case the energy required to disorient the charge carriers and for their subsequent release from the trapping sites is expected to be higher.

The appearance of peaks corresponding to homocharge in case of short-circuit and open-circuit TSC can also be understood in terms of a large amount of homocharge on the surface of the sample, then this homocharge should give rise to a large electric field inside the samples across the thickness. If the temperature is raised to allow the movement of dipoles, this internal field, in the case of open-circuit TSC, would orient the dipoles in the direction of the field. This polarization current is observed in the external circuit and direction of the 
current is the same as that of the neutralizing current of homocharge.

\section{Conclusions}

The systematic analysis of various results have indicated that the polarization phenomena in the present case is due to the existence of heterocharge due to dipole orientation and ionic homocharge drift, together with the injection of charge carriers from electrodes and their subsequent localization in surface and bulk traps. The enhancement of peak current, with increase in PMMA, has been explained on the basis of induced dipoles created because of the piling up of charge carriers at the phase boundary of heterogeneous structure of blend and increase in mobility of charge carriers due to plasticization effects.

\section{References}

Frensch H and Wendorff J H 1986 Polymer 271332

Gubkin A N 1957 Sov. Phys.-Tech. Phys. 21813

Jiska J J, Barlow J W and Paul D R 1981 Polymer 22981

Keller J M, Datt S C, Dubey R, Singh R and Khare P 1993 Phys. Status Solidi 139391

Khare P K 1994 Indian J. Pure \& Appl. Phys. 32160
Khare P K and Srivastava A P 1993 Indian J. Pure \& Appl. Phys. 31126

Khare P K and Singh R 1994 Polym. Int. 34407

Khare P K and Srivastava A P 1994 Indian J. Phys. A68 291

Khare P K, Keller J M and Datt S C 1992 Indian J. Pure \& Appl. Phys. 30768

Khare P K, Surinder P and Srivastava A P 1993 Indian J. Pure \& Appl. Phys. 31126

Khare P K, Chandok R S, Dubey Neeraj and Srivastava A P 1994a Polym. Int. 35153

Khare P K, Keller J M, Gaur M S, Singh R and Datt S C 1994b Polym. Int. 35337

Khare P K, Sandeep Shrivastava and Srivastava A P 1994c Indian J. Phys. A68 129

Khare P K, Keller J M, Gaur M S, Singh R and Datt S r 1996 Polym. Int. 39303

Pillai P K C, Gupta B K and Goel M 1981 J. Polym. Sci. Polym. Phys. 19461

Shrivastava R K, Qureshi M S and Bhatnagar C S $1978 \mathrm{Jpn}$ J. Appl. Phys. 171537

Shrivastava S K, Ranade J D and Srivastava A P 1979 Jpn J. Appl. Phys. 182303

Shrivastava S K, Ranade J D and Srivastava A P 1981 Polymer 221645

Sinha H C, Talwar I M and Stivastava A P 1981 Thin Solid Films 82221

Vanderschueren J and Linkens A 1978 J. Appl. Phys. 494195 